\title{
Studying the Thenar Eminence of Amateur cooKs (STEAK) study: a double-blinded, cross-sectional study
}

teak browning is the result of the protein myoglobin being denatured by heat, and is strongly correlated with heterocyclic amine formation. ${ }^{1}$ Heterocyclic amines are suspected to be a risk factor in colorectal cancer because of their association with oxidative stress, so that overcooked meats may be carcinogenic. ${ }^{2}$ On the other hand, the levels of potentially toxic bacteria, including Campylobacter jejuni, Escherichia coli O157:H7, Salmonella spp. and Listeria monocytogenes, rapidly decline the more a steak is cooked. ${ }^{3}$ At the same time, lean beef has been found to have positive cardiovascular health benefits in that it reduces low-density lipoprotein-cholesterol levels, and should thus not be excluded from a balanced diet. ${ }^{4}$ As a result, the importance of determining the doneness of a steak is not limited to the fancy of gastronomes, but is an important health question.

Several methods have been developed to assess the doneness of a steak, including the invasive techniques of internal steak temperature monitoring and visual assessment. ${ }^{5}$ A third, non-invasive technique is the "finger test", using the thenar eminence of the human hand. The thenar eminence is made up of the abductor pollicis brevis, flexor pollicis brevis and opponens pollicis muscles. This method for determining steak doneness compares the tension of the surface of the steak with that of the thenar eminence while the hand is in different positions. The objective of this study was to determine the accuracy of the finger test.

\section{Methods}

This was a double-blinded, crosssectional study. Ethics approval was obtained from the Monash University Human Research Ethics

Abstract

Objectives: To evaluate the reliability of using the thenar eminence to determine steak doneness.

Design: Double-blinded, cross-sectional study.

Setting: Various home kitchens in Melbourne, Australia.

Participants: Amateur/home cooks.

Main outcome measures: The accuracy of the finger test (the tenseness of the thenar eminence in different hand positions) for determining how well a random beef steak has been cooked (rare $\vee$ medium-rare $\checkmark$ medium $\vee$ well-done). We also examined whether participants improved with practice and whether the accuracy of the finger test was correlated with age, sex, cooking experience or self-rated steak-cooking ability.

Results: Twenty-six participants completed the study, and showed that they could accurately determine the doneness of a steak with the finger test better than chance $\left(\chi^{2}[1, n=156]=9.88 ; P<0.01\right)$. Their overall accuracy, however, was low (36\%). There was no correlation between accuracy in application of the finger test with the other collected participant and steak variables.

Conclusions: The finger test can be used by amateur cooks to determine beef steak doneness. However, the low overall accuracy of the test suggests that more invasive tests are to be recommended for determining steak doneness for its health benefits.

Committee (MUHREC, approval CF15/441 - 2015000216). Our reporting of this research conforms with the STROBE statement on crosssectional studies. ${ }^{6}$

\section{Participant selection}

The researchers advertised the research sessions by word of mouth in Melbourne, Australia. Participants were included in the study if they were over 18 years of age, were not a professional cook or enrolled in a course leading to a qualification as a professional cook, and were able to attend a research session during a 14-week study period.

All participants provided written consent before participating in the study. Participant sex and age were recorded, as well as data on how often the participant cooked a hot meal each week and their self-rated steak-cooking ability (on a scale of 0 , unable to cook steak, to 10 , a master steak cook).
Participants were then provided with written instructions and photographs, and a demonstration of how to conduct the finger test to determine the doneness of the sample steaks. A steak was considered rare if it had the same tenseness as the thenar muscles during a gentle pinch between the thumb and index finger. In a similar manner, a medium-rare, medium or well-cooked steak has the same tenseness as the thenar muscles during a gentle pinch between the thumb and the middle, ring or little fingers respectively (Box 1 ).

\section{Steak preparation}

We used Australian beef porterhouse steaks, purchased from Aldi in packs of four and stored at $4^{\circ} \mathrm{C}$. How well the steak was to be cooked was determined by a computer-based random number generator. Each participant in a research session tested the same six steaks in the same order. We collected data on steak weight, cooking time and internal 
1 Hand positions for determining the doneness of steak using the thenar eminence: A, raw; B, rare; C, medium-rare; D, medium; $\mathrm{E}$, well-done
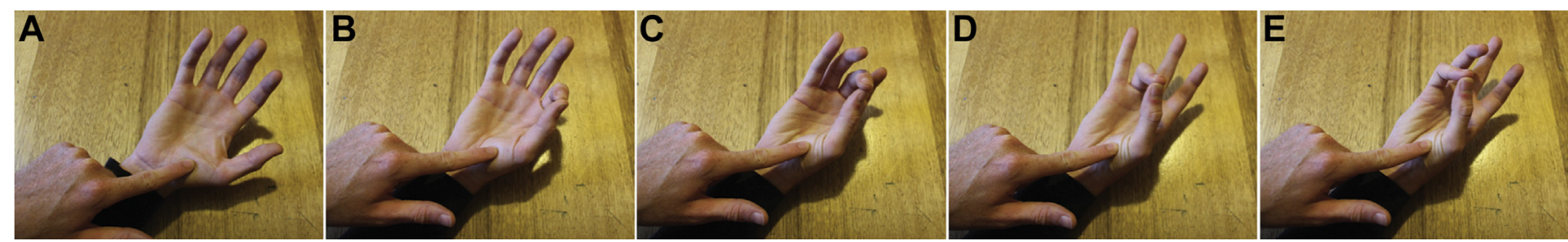

temperature immediately before and after cooking.

All steaks were cooked by one of the authors (TV) in a Crofton non-stick cooking pan (Aldi). The stoves used included gas burner and inductionheated models. Oil and seasoning were not used during cooking. Steak doneness was monitored by assessing the internal steak temperature during cooking, recorded by a wireless grilling thermometer (Bar B Chek, model ET-2213AU, Maverick Industries) with the temperature skewer passing through the long axis of the steak. Steaks were cooked to $40^{\circ} \mathrm{C}$ and then turned onto the uncooked side. After the steak reached the predetermined temperature, it was removed from the pan. A rare steak was cooked to $53^{\circ} \mathrm{C}$, a mediumrare steak to $58^{\circ} \mathrm{C}$, a medium steak to $63^{\circ} \mathrm{C}$, and well-done steak to $75^{\circ} \mathrm{C}$. All steaks rested for at least 2 minutes before being tested by a participant.

\section{Data collection}

Participants were isolated before being asked to sequentially estimate the doneness of three steaks using the finger test. After each participant had rated the first three steaks (preresults), all participants were provided with written feedback on how well each steak had been cooked and how this compared with the participant's estimates. Participants were then isolated again and asked to sequentially rate three more steaks using the finger test (post-results). Participants were given one steak at a time by the supervising researcher, and were not allowed to alter their response after moving on to the next steak. The supervising researcher and participant were both blinded as to how well the steak had been cooked. All steaks were presented with the first cooked surface face-up to minimise visual cues that may have confounded results.

\section{Outcomes}

Our primary aim was to determine whether participants could estimate, better than chance, how well a steak had been cooked, and whether their estimates improved with experience.

Our secondary aims were to determine whether a participant's estimates were correlated with their age, sex, cooking experience or self-rated steak-cooking ability. We also examined steak-related variables, including weight and total cooking time, and whether participants generally overor underestimated how well the steaks had been cooked.

\section{Statistical methods}

For our primary outcome, we used a $\chi^{2}$ goodness-of-fit test to assess whether participants successfully estimated steak doneness more frequently than would be expected by chance $(25 \%)$. We compared preresult and post-result outcomes using the McNemar test. We assumed a Gaussian distribution for our participants' demographic characteristic and the steak-related variables, and therefore used Pearson correlation coefficients to quantify the correlation between these variables and the proportion of successful estimates.

After collecting the data, we decided to also evaluate whether participants repeatedly under- or overestimated the doneness of the tested steaks. To do this, we assigned values of -3 to +3 to each estimate, expressing the relationship of the estimated doneness with our internal control (eg, an estimate of well-done for a steak that was cooked medium was scored as +1 ). We then used a one-sample $t$ test to compare pre- and post-results with a theoretical mean of 0 (ie, no difference), and compared pre-result and post-result differences with unpaired $t$ tests.

All analyses were performed with GraphPad PRISM (version 6.0g, GraphPad Software); $P<0.05$ (twotailed) was defined as statistically significant.

\section{Results}

\section{Participants}

We recruited 27 participants, but one was unable to commence data collection and was excluded from the final analysis. Each participant assessed the doneness of six steaks, resulting in 156 data points. Of our 26 participants, 10 were men (38\%) and the median age was 26 years (range, 24-79 years); they each cooked a median of three hot meals per week (range, 0-7) and their median selfrated steak-cooking ability was 5 out of 10 (range, $0-10$ ).

\section{Primary outcomes}

Participant accuracy in determining steak doneness is summarised in Box 2. For the pre-result assessments, participants did not correctly estimate the doneness of steaks more frequently than by chance $\left(\chi^{2}[1\right.$, $n=78]=2.07 ; P=0.15)$, but were able to estimate doneness better than chance in the post-result stage $\left(\chi^{2}[1, n=78]=9.04 ; \quad P<0.01\right)$; the same applied to the overall results $\left(\chi^{2}[1, n=156)=9.88, P<0.01\right)$. The McNemar test indicated there was no significant improvement between 
2 Accuracy of steak doneness assessment by the 26 participants

\begin{tabular}{lccc} 
Result of assessment & Pre-test & Post-test & Overall \\
\hline Incorrect & 53 & 47 & $100(64 \%)$ \\
Correct & 25 & 31 & $56(36 \%)$ \\
\hline Total & 78 & 78 & $156(100 \%)$
\end{tabular}

3 Correlation of demographic characteristics of participants with the their proportions of correct estimates of steak doneness

\begin{tabular}{lcc} 
& Pearson $\mathbf{r}$ & $P$ \\
\hline Participant demographics & & \\
Sex & 0.28 & 0.16 \\
Age & 0.02 & 0.94 \\
Hot meals per week & 0.02 & 0.93 \\
Self-rated steak-cooking ability & 0.03 & 0.90 \\
Steak variables & & \\
Steak weight & 0.21 & 0.32 \\
Steak doneness & -0.30 & 0.15
\end{tabular}

pre- and post-result assessments $(P=0.14)$.

\section{Secondary outcomes}

Correlations of the accuracy in determining steak doneness using the finger test with sex, age, how many times a week a person prepared a hot meal, and the participant's self-rated steak-cooking ability were not statistically significant (Box 3). The steak's initial weight and cooked doneness (rare $\mathrm{v}$ medium-rare $\mathrm{v}$ medium $\mathrm{v}$ well-done) were also not statistically correlated with the participant's probability of correctly estimating doneness (Box 3).

Participants underestimated the doneness of pre-result steaks by an average of 0.56 points $(95 \% \mathrm{CI},-0.85$ to -0.28 points; $P<0.001)$ and postresult steaks by 0.08 points $(95 \% \mathrm{CI}$, -0.36 to +0.21 points; $P=0.60)$. The pre-result versus post-result finger test difference was +0.49 points $(95 \%$ $\mathrm{CI},+0.08$ to +0.89 points; $P<0.05)$.

\section{Discussion}

\section{Key results}

Participants in our study were able to use the finger test to determine, better than chance, how well a steak had been cooked. There was a trend to improvement with practice, as shown by the difference between the pre- and post-result assessments, but this difference was not statistically significant. We did not identify any participant demographic characteristics or steak variables that were correlated with greater accuracy in using the finger test.

Although participants underestimated the doneness of the steaks by 0.56 points during the pre-result stage, this difference did not translate practically into a difference from actual doneness by a whole number interval. The $95 \%$ CI for this calculation did not include -1.00 , so that it is unlikely that participants were routinely underestimating steak doneness during this stage.

While participants were able to use the finger test to improve the probability that they could determine how well their steak had been cooked, an overall accuracy of only $36 \%$ (56 of 156 assessments) shows that its practical application is likely to be limited. In particular, we recommend against readers using the finger test to determine the doneness of steaks for the purpose of returning the steak to the cook for further preparation. In such cases, the reader might find their steak returned, the degree of doneness unchanged, but the steak newly marinated in excess juices from the cook's anger-provoked sialorrhea.

\section{Limitations}

Most of our participants (23 of 26, $88 \%$ ) were 30 years old or younger. A broader range of ages, particularly staggered towards older participants, might find a different outcome, as older participants are likely to have cooked, on average, many more steaks in their lifetime, a factor that would not have been captured with significance in our analysis.

Many participants in our study commented that it was difficult to determine how well the steak had been cooked because they felt different degrees of doneness in different parts of the steak. Participants were uniformly asked to provide their best estimate using the finger test, but uncontrolled variables, particularly fat content and its distribution, may have reduced the sensitivity of the finger test.

\section{Recommendations}

The finger test has shown a small benefit for amateur cooks, and future research should look at its applicability to other types of meat (eg, pork or lamb) and cooking techniques (eg, boiling or grilling).

Given the results of our study, we suggest that amateur cooks and those wishing to reduce their risk of acute food poisoning or potential carcinogen intake continue to use the invasive tests (ie, internal steak temperature or visual assessment) to determine steak doneness.

Acknowledgements: We would like to thank Melissa Drentin for her assistance in conducting the study.

Competing interests: No relevant disclosures.

(c) 2015 AMPCo Pty Ltd. Produced with Elsevier B.V. All rights reserved.

References are available online at www.mja.com.au. 
1 Alaejos MS, González V, Afonso AM. Exposure to heterocyclic aromatic amines from the consumption of cooked red meat and its effect on human cancer risk: a review. Food Addit Contam Part A Chem Anal Control Expo Risk Assess 2008; 25: 2-24.

2 Figg WD. How do you want your steak prepared? The impact of meat consumption and preparation on prostate cancer. Cancer Biol Ther 2012; 13: 1141-1142.
3 Lahou E, Wang X, De Boeck E, et al. Effectiveness of inactivation of foodborne pathogens during simulated home pan frying of steak, hamburger or meat strips. Int J Food Microbiol 2015; 206: 118-129.

4 Roussell M, Hill A, Gaugler T, et al. Beef in an optimal lean diet study: effects on lipids, lipoproteins, and apolipoproteins. Am J Clin Nutr 2011; 95: 9-16.
5 Chan SH, Moss BW, Farmer LJ, et al. Comparison of consumer perception and acceptability for steaks cooked to different endpoints: validation of photographic approach. Food Chem 2013; 136: 1597-1602.

6 von Elm E, Altman D, Egger M, et al. The Strengthening the Reporting of Observational Studies in Epidemiology (STROBE) statement: guidelines for reporting observational studies. PLOS Med 2007; 4: e296. 